\title{
Do Cultural Beliefs and Practices Influence Place of Delivery among Women? A case of Ibanda district, Uganda.
}

\author{
James Ntozi and Felece Katusiime-Kabazeyo
}

\author{
Department of Population Studies, \\ Makerere University, Uganda. \\ intozi22@gmail.com \\ kkfelece@gmail.com
}

\begin{abstract}
The paper investigates cultural beliefs and practices that influence women's choice of the place of delivery in Ibanda district of Uganda. Primary survey data on I44 women aged 20-49 years, 10 traditional birth attendants (TBAs) and 5 focus group discussions were used in the analysis. Results showed that $55 \%$ of the women delivered from home. Cultural beliefs and practices in the area included use of herbs, burial of placenta, compression of womb, unexposed private parts and delivering alone, but did not determine place of delivery. Multivariate analysis found that level of education and type of housing were the most influential variables. Qualitative data identified reasons for rural women preferring home as a place of delivery including home convenience, family support, rude behaviour by health workers, past positive experience with home delivery, quick labour progression and preference for TBAs. Train current TBAs to recognize and refer risky pregnancies to health facilities.
\end{abstract}

Keywords: Place of delivery, cultural beliefs and practices, influence, women, Uganda.

\section{Introduction}

While motherhood is a positive and fulfilling experience, for many women in Africa, it is associated with suffering, ill health and even death brought by complications during pregnancy and childbirth (Urassa, Massawe, Lindmark, \& Nystrom, 1997, United Nations Children Fund (UNICEF) 2009). In low and middle-income countries, deliveries still occur at home and without assistance of trained attendants (Titaley, Hunter, Dibley and Heywood, 2010). This has generated serious concern, since any births require an appropriate care. Women normally turn to traditional birth attendants (TBAs) because other health workers are not available, are too expensive or because TBAs understand the culture and respect women's needs during delivery (Yakong, Rush, Bassett-Smith Bottorf and Robinson, 2010). However, it has been observed that even trained TBAs cannot in most cases, save women's lives because they are unable to detect or treat complications and are often unwilling to refer these cases to modern health facilities for better management (Carlough \& McCall, 2005). This has contributed to the slow decline of the world maternal mortality (Hogan, et.al. 20I0).

In Uganda, maternal mortality is still high and declines slowly. In 2011, maternal mortality was estimated at $438 / 100000$ live births, which approximates at 16 women dying every day from pregnancy and childbirth related causes. In southwest Uganda, only 40 percent of the deliveries took place at health facilities compared to 57 percent that took place at home (Uganda Bureau of Statistics (UBOS) and ICF International Inc 20I2).

There have been several attempts to increase institutional deliveries in Uganda (EkirapaKiracho, Waiswa, Rahman, Makumbi, Kiwanuka, et.al. 20 I I) These include training of midwives in safe motherhood and lifesaving skills; construction of level IV health centers (with operating theatres and at least a doctor to man it) in every county to offer emergency obstetric care; and training of comprehensive nurses who can offer both midwifery and nursing. However, in southwest Uganda, the proportion of mothers delivering in health facilities continued being low (22\% in 1995, $22 \%$ in 2001 , $31 \%$ in 2006 and $40 \%$ in 201 I), but the rates are even lower in rural areas and among the poorest quintile of the population (UBOS and Macro International Inc 2007, UBOS and ICF International Inc 20I2).

Since the factors influencing the place of delivery are many, this study focuses on traditional cultural beliefs and practices related to childbirth that people believe in and their significance to influence choice of place of delivery among rural women in Uganda, which are not well explored in the existing literature. 
This information should give a better understanding of the science behind these traditional beliefs and practices and if possible be incorporated into the health intervention programmes to increase utilization of maternal health services and reduce the high maternal mortality rate within Uganda.

The main objective of the study was to establish reasons why women opt for home delivery in rural Uganda. Specifically, the study was to identify various traditional beliefs and practices related to place of delivery, assess their contribution in influencing the place of delivery and examine the relationship between socio-economic and demographic variables and place of delivery.

\section{Literature Review and Theoretical Framework}

Place of child birth is influenced by various cultural and other factors. In Ethiopia, Shiferaw, et.al., (2013) found that a significant majority $(78 \%)$ of mothers was attended by traditional birth attendants in a home, because the respondents believed that it was not necessary $(42 \%)$ and not customary $(36 \%)$ to be delivered in modern health facilities. Traditional birth attendants were seen as culturally acceptable and competent health workers. Women who had delivered at modern health facilities had reported poor quality of care and negative experiences. In addition, women had low awareness on the advantages of skilled attendance at delivery.

In Zambia, it was found that traditional birth assistants (mbusas) advised childbearing women on appropriate cultural childbirth practices and assisted with deliveries at home. They also advised women on the use of traditional medicine, for example, to widen the birth canal and to precipitate labour. If something went wrong during labour, they relied on traditional beliefs and witchcraft to explain the mishap and expected the woman in labour to confess her purported 'bad' behaviour (Maimbolwa et. al. 2003). The authors concluded that these social support women, including those who considered themselves as mbusas, lacked understanding of the causes of obstetric complications during childbirth, and had inadequate knowledge of the appropriate management of labour.

An important factor that prevents women from delivering at health facilities is that they do not want to expose their private parts to strangers. In Uganda, it is uncommon to see the private parts of a woman even on genuine health reasons. Many women would rather die than expose their genital parts. Kyomuhendo, (2003), in her study in Uganda found that women were not willing to expose their private parts to the male gynecological doctors, midwives and nurses some of whom were young to be their daughters.
Many people and cultures in Uganda do not regard childbirth as a condition, which requires medical care by a professional doctor/ health worker because women culturally are expected to be brave and strong while in labour (UBOS and Macro International Inc 2007). In addition, it was found that although women preferred to deliver in health facilities, most do not do so because they cannot afford the cost of drugs and supplies which are demanded in a situation of poverty and limited male support (Waiswa et al. 2008).

In Benin, women do not visit maternity facilities because they take pride in giving birth without assistance. According to them, childbirth is an opportunity for a woman to show courage and bring honor to her and the husband's family. It was found that some women hide themselves while in labour, and even cut umbilical cords themselves (Sargent, 1990).

In other parts of West Africa, research found that, women in Sokoto and Zaria in Nigeria, and among Malians living in Accra, who had difficulty in delivering vaginally at home were reluctant to go to the hospital for fear of being operated on and hence be stigmatized in their community. Societal expectations are that a woman who does not deliver vaginally has failed in her essential role (Leslie, 1992).

A study of Southern Tanzania by Mrisho, et. al. (2007) found that issues of cultures, tradition and lack of privacy were among the key determinants of the place of delivery. Other factors were lack of money, lack of transport, sudden onset of labour, short labour, staff attitudes and the pattern of decisionmaking power within the household. There were substantial variations between ethnic groups $(p<0.0001)$, headship of households where women lived, level of women's education, age of women and household poverty levels.

A study of 6 African countries by Stephenson, et.al. (2006) found that maternal age, parity, educational status, religion, and marital status were all influential in a woman's decision to deliver her last child in a health facility or home, although the pattern and magnitude of these relationships varied across the 6 countries, indicating geographic and cultural variations in the pathways through which these variables influence health behavior. The significance of urban residence in 4 countries highlights the benefits of greater service availability afforded to urban residents. Two variables were consistently related to the decision to deliver a child in a health facility in all 6 countries: receiving prenatal care and delivering a previous child in a health facility. The latter demonstrates a simple relationship: women who have delivered a child in a health facility are the most likely to continue to deliver future children in 
health facilities, irrespective of maternal age and parity.

Chandrashekhar, Joshi, Sreekumaran, Giri and Chuni (2006) observed that among urban 240 mothers in Western Nepal, most of them planned home deliveries $(58.3 \%)$, and only $6.2 \%$ of deliveries had a skilled birth attendant present, while a sizeable proportion of mothers (15.8\%) gave birth alone. The main reasons cited for delivering at home were 'preference' (25.7\%), 'ease and convenience' (21.4\%) for planned deliveries while 'precipitate labor' (5।\%), 'lack of transportation' (I8\%) and 'lack of escort' during labor $(11 \%)$ were cited for the unplanned ones. Unfortunately, the main dangers found in Nepal case were: only $16.2 \%$ of women had used a clean home delivery kit and only $38.3 \%$ of birth attendants had washed their hands. The umbilical cord was cut after expulsion of placenta in $64.2 \%$ of deliveries, fortunately a cord was cut using a new/boiled blade in $90.4 \%$ of deliveries.

Theoretically, the place of delivery is influenced indirectly by the background factors of women and directly by intermediate variables. The background factors include age, residence, marital status, education and occupation of women. For example, older women may utilize health services more than the younger ones because they know the dangers of unsupervised deliveries (Babalola \& Fatusi 2009). On the other hand, married people tend to utilize health services more than any other category (Rosenstock 2005) while educated mothers easily use and interpret health messages than uneducated and mothers with some form of occupation find it less difficult to use health services because they can afford to pay for them (Reynolds, Wong \& Tucker, 2006). Education is the only individual-level variable that is consistently a significant predictor of service utilization, while socio-economic level is a consistent significant predictor at the household level (Babalola \& Fatusi, 2009).

In most cases, the background factors go through intermediate variables like accessibility to and affordability of place of delivery. Distance and transport issues in rural areas are highly significant factors affecting women's access to health services, especially emergency care (Say \& Raine, 2007). Even if women do attempt to get to hospital for delivery, they may arrive too late for their lives to be saved.

Being poor limits access to information and appropriate healthcare, which poses major challenges to determining place to deliver in. Poverty can hinder utilization of skilled delivery even after controlling other potential confounders such as age and marital status, the poor can hardly afford user fees and other mandatory requirements such as cotton, gauze and child clothing (Gage 2007). This variable can facilitate background factors or can directly influence place of delivery.

However, cultural beliefs and practices are also important in influencing the place of delivery. These are cultural-related norms that people believe in which negatively or positively influence the choice on the place of delivery. They often lead to self-care, home remedies, consultation with TBAs and advice of older women in the house, which cannot be ignored (Mrisho et al. 2007). Cultural factors directly influence place of delivery.

\section{Data and Methods}

The study was cross-sectional, using both quantitative and qualitative approaches. Primary quantitative data was used and was collected in a survey conducted among women in the reproductive age of 20 and 49 years. The younger women below 20 years were excluded because most of them had not given birth and they did not understand the cultural beliefs and practices, which were the focus of the study. The qualitative study probed traditional birth attendants (TBAs) who were 40-49 years as key informants and Focused Group Discussions (FGDs). The study targeted only women who had ever given birth.

At the time of this study, Ibanda was one of 112 districts of Uganda situated in rural southwest of the country, just south of the equator. The district was selected because it is rural and remote, about 300 kilometres away from Kampala, the metropolitan and capital city of Uganda, is about 25 kilometres from Ibanda town council, has no borders with outside countries and no study on maternal issues had been conducted before. In the past, it was part of Ankole region, which was found to have high fertility rate (Ntozi, 1995) and high maternal mortality levels (UBOS and Macro international inc. 2007). The area of study - Nyarukiika in Rukiri subcounty, was far (about $20 \mathrm{kms}$ ) from the Ruhoko Health centre with antenatal facilities. Much further (about $25 \mathrm{kms}$ ) was a Catholic missionary hospital - Kagongo with better maternal facilities than Ruhoko health centre.

Using simple random method, Rukiri subcounty was selected out of 7 sub-counties of rural lbanda district. Out of five parishes of Rukiri subcounty, Nyarukiika parish was chosen through simple random method. This parish had II villages with a total of 225 women aged 20-49 years old. A table of random numbers was used to select I44 out of 225 women. Although all eligible women (225) in the selected parish should have been interviewed, it was not possible due to financial reasons. Hence a sample size of I44 was determined using the Israel (2009) formula, when the target women were 225 and level of precision was $5 \%$. 
Quantitative data was collected from the selected I 44 women using a structured questionnaire with both open-ended and close-ended questions. Trained interviewers were used to administer the questionnaire supervised by the researchers.

After the field, data was coded and edited. The program Epidata was used to enter the data into the computer, thereafter it was imported into STATA (8.2) for further analysis, which was done at three levels: univariate, bivariate and multivariate.

At the univariate and bivariate levels, descriptive statistics and frequency tables were generated in order to summarize the data. Frequency and percent distributions were run to give information on the influence of cultural beliefs and practices and socioeconomic and demographic factors on influencing place of delivery.

Multivariate analysis was done for further statistical analysis. The binary logistic regression model was fitted to establish whether the independent variables could significantly explain the influence of culture on the place of delivery among women in Rukiri sub-county. This model was used because the dependent variable in the study is dichotomous, that is, a woman delivers from a health facility $=$ I or from home $/$ TBA $=0$.

The model can be expressed as follows:

$$
\log \left[\frac{p_{i}}{1-p_{i}}\right]=b_{0}+b_{1} x_{1}+b_{2} x_{2}+\ldots \ldots . b_{k} x_{k}+e_{i}
$$

Where,

$p_{i}$ is the probability that a woman delivers from a health facility

$b_{0}$ is the coefficient of the constant

$b_{i}$ are the regression parameter estimates

$x_{i}$ are independent variables.

$e_{i} \quad$ is the error term.

The dependent variable was the place of delivery which is binary (delivering from health facility or not). The independent variables were cultural, socio economic and demographic factors, which included mother's education, age, occupation and marital status and type of house lived in, as well as practice of traditional beliefs.
Regarding the qualitative data, traditional birth attendants were purposively selected basing on their age and experience. Older and experienced women tend to be more knowledgeable in cultural and maternal related issues than the younger and unexperienced ones. The study used TBAs of ages 40-49 and with an experience of more than 5 years. Ten TBAs in the whole parish were subjected to the interview as key informants. In addition, five FGDs for women of various reproductive age groups, each with six mothers were interviewed.

An interview guide was used to collect data from the TBAs and a focus group discussion guide employed to talk about various issues in the focus group discussion sessions. Both tools were implemented by one of the researchers helped by a note taker.

The data was analyzed using a thematic approach whereby in collecting data, patterns of experience were listed from direct quotes and paraphrased in common ideas in the interview. Then the researchers identified all data that related to the already classified pattern and combined them into subthemes. It is from this that arguments were built, leading to conclusions and recommendations (Constas, 1992). Concurrent triangulation design was applied in analyzing and presenting quantitative and qualitative data.

This study was approved by Makerere University organs and the National Council for Technology and Science of Uganda. In the field, the district and subcounty authorities approved the research and all participants gave their consent to be interviewed.

\section{Results}

Table I shows the distribution of respondents by background characteristics. Of the 144 women, over a third were young aged 20-29, close to half were middle aged and less than a fifth were old. Almost all of them were married with a tiny proportion divorced/separated. Close to two thirds of the women were involved in farming activities, while less than a fifth were in business/trade and one in 12 were public servants. In terms of education, over 3 in 5 had stopped in primary school, while I in 5 had acquired at least some secondary level education and less than I in 6 did not go to school. Half of the women lived in semi-permanent houses, $30 \%$ stayed in permanent houses and $18 \%$ were living in temporary structures. 
Table I: Distribution of women by background characteristics

$\begin{array}{lll}\text { Characteristics } & \text { Frequency } & \text { Percen } \\ \text { Age } & & \\ \text { 20-29 years } & 52 & 36.1 \\ \begin{array}{l}30-39 \text { years } \\ \text { 40-49 years }\end{array} & 67 & 46.5 \\ \text { Marital status } & 25 & 17.4 \\ \text { Married } & 137 & 95.1 \\ \text { Divorced/ Separated } & 7 & 4.9 \\ \text { Occupation } & & \\ \text { Agriculture/Farming } & 91 & 63.2 \\ \text { Business/trade } & 25 & 17.4 \\ \text { Public servant } & 12 & 8.3 \\ \text { Not working } & 16 & 11.1 \\ \text { Education } & & \\ \text { No education } & 22 & 15.3 \\ \text { Primary } & 91 & 63.2 \\ \text { Secondary }+ & 31 & 21.5 \\ \text { Type of the housing } & & \\ \text { Temporary } & 25 & 17.4 \\ \text { Semi-permanent } & 75 & 52.1 \\ \text { Permanent } & 44 & 30.5 \\ \text { Total } & 144 & 100.0\end{array}$

Table 2 shows the distribution of women by place of delivery, traditional beliefs and practices and the type of delivery attendance. The table indicates that over half of the respondents delivered their last baby from their homes assisted by traditional birth attendants, mother-in-law, and other non-medically trained people, while only 45 percent delivered in a modern health facility being helped by medically trained personnel. Three in ten women practiced traditional rituals during the last birth. Most of the respondents were aware that beliefs and rituals existed but this did not necessarily mean that whoever knew the rituals practiced them and even a few that applied and respected them reported that beliefs and rituals could not stop them from delivering from the health facilities.

The reported traditional beliefs and practices included use of herbs, burial of placenta, womb compressing, not exposing the private parts and delivering alone. The most common practice was use

of herbs. During labor, TBAs, women's mothers and relatives applied herbal medicine on the abdomen of the mother. The clay capsule (emumba), were a mixture of clay soil with different herbs to soften the pelvis and the woman would deliver sooner. Tea leaves to quicken labour progression were also given. Secondly, women compressed their wombs after delivery in order to keep them in shape and stay beautiful. In the health facilities, this would not be allowed as it is dangerous and could dislocate women's bones. Thirdly, the respondents believed that it was not proper for a woman to expose her private parts to strangers, like health workers, some of whom were men. Fourthly, it was also believed that courageous and strong women delivered alone without any assistance, which is very risky to the health of the mother. 
Table 2: Distribution of women by place of delivery, traditional beliefs and practices and the type of delivery attendance.

Place of delivery for the last birth

TBA/Home

$\mathrm{HC}$

Practice of traditional beliefs on last birth

No

Yes

Beliefs and practices

Use of herbs

Placenta burial

Compressing the womb

Non exposure of private parts to strangers

Delivering alone

Don't know

Who attended to you?

Health personnel

TBA

Mother-in-law

None

Husband

Total

Table 3 gives results from applying binary logistic regression on the data linking place of delivery and women's characteristics, in order to estimate predictors of place of delivery. In the table, there were two predictors, namely, women's education and economic status of the household. Women with at least secondary education had almost 8 times more chances of delivering in modern health facilities than uneducated women (OR $=7.6, p=0.035)$. This is an expected finding in that women with high education had more information than others to help her appreciate the advantages of modern health facilities over home delivery. Similar results were found by studies done in Turkey by Celik and Hotchkiss, (2000), in Enugu, Nigeria by lkeako, Onah and Iloabachie, (2006) and in Lao by National Statistical Centre, (2007).

According to Table 3, the second predictor of place of delivery is type of house a woman lived in, which was used in this study as a proxy for economic status. The women who lived in permanent houses were considered to be rich, while those who stayed in temporary building structure were poor and those

$\begin{array}{rr} & \text { Frequency, } \mathbf{n}=144 \\ 79 & 54.9 \\ 65 & 45.1 \\ 101 & 70.1 \\ 43 & 29.9 \\ & \\ 66 & 45.8 \\ 24 & 16.7 \\ 20 & 13.8 \\ 15 & 10.5 \\ 11 & 7.6 \\ 8 & 5.6 \\ & \\ 65 & 45.1 \\ 56 & 38.8 \\ 9 & 6.3 \\ 9 & 6.3 \\ 5 & 3.5 \\ 144 & 100.0\end{array}$

in semi-permanent houses were medium rich. The results in the table show that respondents who lived in permanent houses had close to 9 times more chances of delivering from a modern health facility than those who stayed in temporary structures $(O R=8.8, p=0.003)$. This is because women from rich households could afford to transport themselves and the cost of delivering in modern health facility, compared to the poor women. Previous studies confirmed that women from low economic status find it difficult to use modern health facilities for delivery in rural Tanzania (Mrisho et al., 2007) and in Ethiopia (Warren 2010). Also in Nepal, women with higher income levels gave birth in a hospital compared with those with lower income (Pradhan, 2005).

It is important to note from Table 3 that the practice of traditional beliefs was not a predictor of place of delivery. This implies that these beliefs and practices have lost influence, may be as a result of education and modernization. In fact, the odds ratio of women not practicing the traditional beliefs and values using modern health facility was 2.2 times those practicing the beliefs. 
Table 3: Results of Binary Logistic Regression Model

\begin{tabular}{|c|c|c|c|c|c|}
\hline $\begin{array}{l}\text { Variable } \\
\text { Age }\end{array}$ & Odds Ratio & Std. Err. & $\mathrm{p}>\mathrm{z}$ & \multicolumn{2}{|c|}{ 95\% Confidence Interval } \\
\hline $20-29 *$ & 1.000 & -- & -- & -- & -- \\
\hline $30-39$ & 0.558 & 0.276 & 0.239 & 0.211 & 1.474 \\
\hline $40-49$ & 0.704 & 0.463 & 0.594 & 0.194 & 2.555 \\
\hline \multicolumn{6}{|l|}{ Marital status } \\
\hline Married* & 1.000 & -- & -- & -- & -- \\
\hline Divorced/ Separated & 0.629 & 0.656 & 0.657 & 0.082 & 4.861 \\
\hline \multicolumn{6}{|l|}{ Education } \\
\hline No education* & 1.000 & & & & \\
\hline Primary & 2.382 & 1.822 & 0.256 & 0.532 & 10.665 \\
\hline Secondary + & 7.641 & 7.373 & 0.035 & 1.153 & 50.639 \\
\hline \multicolumn{5}{|l|}{ Occupation } & \\
\hline Business/trade & 2.724 & 2.675 & 0.308 & 0.397 & 18.670 \\
\hline Agriculture & 2.787 & 2.357 & 0.226 & 0.531 & 14.626 \\
\hline \multicolumn{6}{|l|}{ Type of house } \\
\hline Temporary* & 1.000 & -- & -- & -- & \\
\hline Semi-permanent & 0.633 & 0.362 & 0.424 & 0.207 & 1.939 \\
\hline Permanent & 8.812 & 6.359 & 0.003 & 2.142 & 36.250 \\
\hline \multicolumn{6}{|l|}{$\begin{array}{l}\text { Practice of traditional } \\
\text { beliefs }\end{array}$} \\
\hline Yes* & 1.000 & -- & -- & -- & \\
\hline No & 2.233 & 1.159 & 0.122 & 0.808 & 6.176 \\
\hline
\end{tabular}

*Reference category

Reasons for preferring home delivery to modern health facility.

To explain the continued preference of home to modern health facility, the study collected qualitative data from the community. The community responses on why they preferred giving birth at home to delivering at modern health facilities were several fold. First, the community reported that delivering at home was easy and convenient because the women did not have to move from one place to another. Delivering at home would ensure that the women would not give birth on the way to a modern facility, which was believed to be a taboo, making children born in such situation never to settle at home as they grow up. A focus group discussion member aged 39 years said:

"I would like to deliver at home because it is easy and convenient. Engaging in unnecessary movements in the labour process may be dangerous and fatal, some women sit on their babies while on the way to the hospital and the children die. In addition children born while on the journey are ever moving here and there".

Similar reasoning was found in a study conducted in Philippines by Schwartz et. al (1993), with a result that giving birth at home would avoid unnecessary movements of heavily pregnant women.

A second reason was that women delivering at home get family support. The presence of family members such as the women's mother, mother-inlaw, husband and other relatives provides the needed psychological support and physical touch like a back massage and gentle touching of the abdomen. A 25year woman in a FGD stated:

"I would like to deliver at home because I want my husband to stay with me during labor so that I feel his warmth and love. My husband would hold my hands, and the TBA would stay with me all the time, but in the hospital, the midwife predicts the time for delivery and goes away".

A study by Moscucci (2002) similarly found that women in Britain delivered from their homes because they preferred their husbands and other 
close family members to take care of them and provide some psychological and emotional support.

In contrast to the family support, most women complained of being treated as strangers in the modern health facilities.

"I prefer giving birth at home, assisted by TBA or relatives rather than in the unfamiliar setting of a health centre $(H C)$. We the uneducated have problems. Even before you talk, the midwife thinks automatically that you do not understand. I was abused at HC that I did not appear in good clothing and had no new clothes for the baby. Moreover, I had taken old clothing I used for the older children although they were old, they were not torn. I was embarrassed! Health workers only like the rich" (40years- old woman respondent).

It was reported that women are made to feel powerless by the health workers. Sometimes midwives are overworked and too tired to listen to the patients and end up being rude and unfriendly. Although rural women admitted that they were poor, it was not the responsibility of a midwife to inform them of their poverty. In Ghana, women were discouraged from delivering at modern health facility, due to abusive language and lack of tolerance by health workers. (D'Ambruoso et al., 2005).

Furthermore, when women are having labour pains, some of them make noise and others become weird. In response, family members calm them down with sympathetic words and encouragement, unlike in health facilities where noise means that you are disturbing others and becoming unruly.

"When you go to deliver in a hospital, midwives cannot sympathize with you. When you feel pain and make noise, it is a crime. The midwives can decide to ignore you. In such a situation, I was about to lose my baby because I was ignored and when I called health workers for help, they started laughing at me, but God helped me and they came when they saw the baby had come out" (30-years-old woman).

This makes the women regret why they went to the modern health facilities for delivery. In Nigeria women too delivered from home because of unfriendly behavior of medical staff (Envuladu et al., 2003).

Another reason for preferring delivering a baby at home to a modern health facility was to ensure privacy. Women in this society fear being naked and grew up knowing that their naked bodies belonged to their husbands only, but not strangers. When delivering their babies, women try to maximize privacy and this was easier at home. In contrast, the environment in which women delivered at modern health facilities lacked privacy. Often the labour ward at the health facility was crowded by pregnant women and their relatives and there are no special rooms for delivering babies. A 44 year old woman lamented:

"Imagine a midwife fit to be your daughter directing you with pride. Can you imagine adding pain on an already existing pain? Exposing my private parts to my daughter is an abomination in my tribe".

Mrisho et. al. (2007) found that women in rural Tanzania were afraid to be seen delivering babies in public. In addition, where some hospitals train midwives, some women who come to deliver become case studies for students, which women do not like.

Previous experience was taken into account when selecting the place of delivery. Most women in FGDs reported that they had some experiences delivering at home from their first child to their current pregnancies. If the previous place of delivery was good, it was more likely to use the same place in their next births. In addition, the childbirth experiences of their mothers, mothers-in-law, aunts and grandmothers influenced where their deliveries took place.

"If everyone in our family has been delivering here safely, then why waste time going to the hospital, what if something bad happens to me in the hospital? Most likely one can die because most of the maternal deaths have taken place there" (23-years-old woman).

Similarly, in Ethiopia most women who gave birth from home regarded delivering from formal institutions as not necessary and even not cultural (Warren, 2010, Central Statistical Authority (CSA) [Ethiopia], and ORC Macro, 2006). Ignorance contributes to such perceptions, which require giving education to future young mothers and maternal health sensitization to the current illiterate women.

Women who experience quick progression to labour end up delivering at home. In case of emergency, when there is no warning that labour would start, women in the rural areas without immediate transport, they had no alternative, but to deliver at home.

"Labor was quick and easy, I could not go to the health facility on time. More so because I knew that pregnancy takes a period of nine months but $I$ delivered at eight months, so I was not prepared at all" (20-years-old woman who had delivered).

A study done by Mrisho et al., (2007), in rural Tanzania found that sudden onset of labor forced women to deliver at their homes.

The last reason for preferring home to health facility delivery was preferring assistance from TBAs and being satisfied with their services to those of health workers. Most women in FDGs praised TBAs of being familiar with them, not expensive, being 
experienced, caring and friendly. Traditional birth attendants reported that they were preferred because of their experience. Others said that it is because women cannot afford health services, while others believed that women liked to be assisted by the people they knew and are familiar with. Cost and place of delivery were related as TBAs were mostly rewarded in kind by their clients through gifts and small amounts of money. The most treasured gift was a special meal locally referred to as "Ekihuuro". This was manageable and affordable to the women who were free to prepare what they afforded.

Similarly, in rural Tanzania, women from poor families hardly use health facilities for delivery. They lacked money for transport when the facility is located at a distant place and money to pay for delivery kit, hospital bills as well as food while at health facility and some hospitals could not discharge women with no money until their debts were paid in full (Mrisho et al., 2007).

Also, Kyomuhendo (2003) found that TBAs in Uganda assisted women to deliver, deal with pain and solve minor problems during delivery. They provide care that many women would otherwise not be able to access elsewhere, which increased women's preference for their delivery services. However, the herbs that women use were medically perceived to be harmful to the health of women because they were not recommended and tested, unlike what modern medicine went through before approval for use.

\section{Conclusions}

The study found that there were various cultural beliefs and practices related to childbirth. Although women respected and performed cultural beliefs and practices, the beliefs and practices did not influence their choices on place of delivery. Women's level of education and their economic status influenced their choices on where to deliver their babies. Besides, the qualitative data reported other factors that influenced women's choice of place of delivery and these were, home convenience, family support, rude behaviour by health workers, past positive experience with home delivery, quick labour progression and preference for TBAs.

\section{Recommendations}

To improve maternal healthcare requires that pregnancy and childbirth be conceived as health conditions with potential risks, which require the presence of modern health-care providers. However, TBAs will continue being used in delivery in rural Uganda since the majority of women are demanding their services. This is because most rural women do not have financial ability to deliver from the health facility and can only afford the services of TBAs. Therefore it is necessary to train and use the TBAs so that they can be able to recognize pregnancy complications and refer those with risky conditions, deliver positive messages on benefits of institutional delivery and also conduct deliveries in case of emergency and normal cases.

The only direct significant determinant of women's choice of place of delivery was education. Therefore, the government of Uganda should seriously continue promoting female education and women empowerment and provide regular health education to the current active reproductive women on the dangers of home delivery as a way of reducing maternal mortality in the country.

There is need to do a similar study at the national level to understand different causes of poor utilization of maternal health services particularly the place of delivery and ways of improving them.

\section{Limitation of the study}

Researchers lacked enough money to conduct a large survey, which limited the sample to only I44 respondents, since studies on maternal issues should be large to reduce sampling errors. However, since the study is not about computing maternal mortality rates, the sample is large enough to infer the issues discussed in the study. While the results obtained can represent lbanda district situation, it would be hard to use the results to represent Uganda, since there are many ethnic groups with different cultural practices.

\section{References}

Babalola, S., \& Fatusi, A. (2009). Determinants of use of maternal health services in Nigeria-looking beyond individual and household factors. BMC pregnancy and childbirth, 9(I), I.

Carlough, M., \& McCall, M. (2005). Skilled birth attendance: What does it mean and how can it be measured? A clinical skills assessment of maternal and child health workers in Nepal. International Journal of Gynecology \& Obstetrics, 89(2), 200208.

Central Statistical Authority (CSA) [Ethiopia], and ORC Macro. (2006). Ethiopia Demographic and Health Survey 2005. Addis Ababa, Ethiopia and Calverton, MD, USA: CSA and ORC Macro.

Celik, Y., \& Hotchkiss, D. R. (2000). The socioeconomic determinants of maternal health care utilization in Turkey. Social Science \& Medicine, 50(I2), I 797-I806.

Chandrashekhar, T., S., Joshi, H. S., Sreekumaran, B. V., Giri, S. and Chuni, N. (2006). Home delivery and newborn care practices among urban 
women in western Nepal: a questionnaire survey. BMC Pregnancy and Childbirth BMC series 6:27.

Constas, M.A. (1992). Qualitative Analysis as a Public Event: The Documentation of Category Development Procedures. American Educational Research Journal, 29(2): 253-266.

D'Ambruoso L, Abbey M. and Hussein J. (2005). Please understand why I cry out in pain: Women's account of maternity services during labour and delivery in Ghana. BMC Public Health 5, 140.

Ekirapa-Kiracho, E. Waiswa, P. Rahman, M H., Makumbi, F., Kiwanuka, N. Okui, O. Rutebemberwa, E. Bua, J. Mutebi, A., Nalwadda, G. Serwadda, D., Pariyo G. W. and Peters D. H. (20I I). Increasing access to institutional deliveries using demand and supply side incentives: early results from a quasi-experimental study. BMC International Health and Human Rights, I I (Suppl I):SI I.

Envuladu E.A, Agbo H.A, Lassa S, Kigbu J.H, Zoakah A. (2003). Factors determining the choice of a place of delivery among pregnant women in Russia village of Jos North, Nigeria: achieving the MDGs 4 and 5. Department of Community Medicine, Jos University Teaching Hospital, Plateau state.

Gage, A. J. (2007). Barriers to the utilization of maternal health care in rural Mali. Social Science and Medicine, 65(8): I666-I 682.

Hogan, M. C., Foreman, K. J., Naghavi, M., Ahn, S. Y., Wang, M., Makela, S. M., et al. (2010).

Maternal mortality for 181 countries, 1980-2008: a systematic analysis of progress towards Millennium Development Goal 5. The Lancet, 375(9726), 1609-1623.

Ikeako L.C, Onah H.E, Iloabachie G.C. (2006). Influence of formal maternal education on the use of maternity services in Enugu, Nigeria. I Obstet Gynaecol 26:30-34.

Kyomuhendo G.B, (2003). Low use of rural maternity services in Uganda: Impact of women's status, traditional beliefs and limited resources. Reproductive health matters 11: 16-26.

Leslie, J. (1992). Women's lives and women's health: using social science research to promote better health for women. Journal of Women's Health, I(4), 307-3I8.

Maimbolwa, M.C Yamba, B. Diwan, V. and RansjöArvidson, A. B. (2003). Cultural childbirth practices and beliefs in Zambia. Journal of Advanced Nursing, 43(3): 263-274.

Moscucci, O. (2002). Holistic obstetrics: The origin of "natural birth" in Britain. Postgraduate Medical Journal, 79(929)। 68-I 73.

Mrisho, M., Schellenberg, J. A., Mushi, A. K., Obrist, B., Mshinda, H., Tanner, M., et al. (2007).
Factors affecting home delivery in rural Tanzania. Tropical Medicine \& International Health, 12(7), 862-872.

National Statistical Centre, (2007). Lao Reproductive Health Survey 2005. Committee for Planning and Investment (CPI).

Ntozi, J.P.M. (1995). High fertility in rural Uganda: the role of socioeconomic and biological factors. Fountain Publishers, Kampala, Uganda.

Pradhan, A. (2005). "Situation of Antenatal Care and Delivery Practices." Kathmandu University Medical Journal (KUMJ) 3(3): 266-70.

Reynolds, H., Wong, E. L. \& Tucker, H. (2006). Adolescents' Use of Maternal and Child Health Services in Developing Countries. International Family Planning Perspectives, 32(I), 6-16.

Rosenstock I.M. (2005). Why people use health services. The Milbank Quarterly 83(4) - online only.

Sargent C (1990). The politics of birth: cultural dimensions of pain, virtue, and control among the Bariba of Benin. Births and power: social change and the politics of reproduction, edited by $W$. Penn Handwerker. Boulder, Colorado, Westview Press, 1990. 69-79.

Say, L. \& Raine, R. (2007). A systematic review of inequalities in the use of maternal health care in developing countries: examining the scale of the problem and the importance of context. Bulletin of the World Health Organization, 85(I0), 812819.

Schwartz J. B, Akin J. S, Popkin B. M. (1993). Economic determinants of demand for modern infant-delivery in low-income countries: The case of the Philippines. In: Mills A, Lee K (eds). Health economics research in developing countries. Oxford and New York: Oxford Medical Publications.

Shiferaw, S., Spigt, M. Godefrooij, M. Melkamu, Y.and Tekie, M. (20/3). Why do women prefer home births in Ethiopia? BMC Pregnancy and Childbirth BMC series 3:5.

Stephenson, R., Baschieri, A. Clements, S. Hennink, M. and Madise, N. (2006).

Contextual Influences on the Use of Health Facilities for Childbirth in Africa. Amerian Journal of Public Health, 96 (I): 84-93.

Titaley, C. R., Hunter, C. L. Dibley, M. J. and Heywood, P. (2010). Why do some women still prefer traditional birth attendants and home delivery? a qualitative study on delivery care services in West Java Province, Indonesia. BMC Pregnancy and Childbirth BMC series: 10:43.

Uganda Bureau of Statistics (UBOS) and ICF International Inc. (20/2). Uganda Demographic and Health Survey 20II. Kampala, Uganda: 
UBOS and Calverton, Maryland: ICF International Inc.

Uganda Bureau of Statistics and Macro International Inc (2007). Key findings from the 2006 UDHS: A Gender Perspective. Uganda and Calverton, Maryland, USA: Uganda Bureau of Statistics and Macro International Inc.Uganda Bureau of Statistics (2006).

United Nations Children Fund (UNICEF) 2009. The state of the world children 2009: maternal and newborn health. UNICEF, New York.

Urassa, E., Massawe, S., Lindmark, G., \& Nystrom, L. (1997). Socio-economic and physical distance to the maternal hospital as predictors for place delivery: an observation study from Nepal. BMC Pregnancy and Childbirth, 4(8).
Waiswa, P., Kemigisa M. Kiguli, J. Naikoba, S. Pariyo G.W. and Peterson S. 2008. Acceptability of evidence-based neonatal care practices in rural Uganda - implications for programming, BMC Pregnancy and Child birth BMC series 8:2I.

Warren, C. (2010). Care seeking for maternal health: Challenges remain for poor women. Ethiopian J. Health Dev., 24(I): 100-104.

Yakong', V. N., Rush', K. L., Bassett-Smith', J., Bottorff', J. L. and Robinson, C. (20I0). Women's experiences of seeking reproductive health care in rural Ghana: challenges for maternal health service utilization. Journal of Advanced Nursing, 66 (I I): 243I-244I. 